\title{
Managing Gastroesophageal Reflux Disease in Primary Care: The Patient Perspective
}

\author{
Harley Liker, MD, MBA, Pali Hungin, MD, and Ingela Wiklund, MSc, PhD
}

Gastroesophageal reflux disease (GERD) is a chronic disease that affects up to $20 \%$ of the population of Western countries and accounts for around 5\% of a primary-care physician's workload. A better understanding of how GERD affects many aspects of patients' lives may aid the management of patients in primary care. We conducted a systematic review of the effect of GERD on health-related quality of life (HRQL) in the primary-care setting and in the community. Validated questionnaires have shown that GERD patients consulting in primary care experience substantial reductions in both physical and psychosocial aspects of HRQL, as well as work productivity. Impairments in HRQL are seen whether or not reflux esophagitis or Barrett's esophagus is present on endoscopy, and are comparable with or worse than those seen in patients with other chronic diseases such as heart disease, diabetes, and cancer. Surveys in primary care and in the community highlight disrupted sleep, reduced concentration at work, and interference with physical activities such as exercise, housework, and gardening. Psychosocial aspects of patient well-being are also impaired, including enjoyment of social gatherings, intimacy, sex, and many individuals with GERD remain worried about the underlying cause of their symptoms. In conclusion, many aspects of HRQL are impaired in GERD patients. The primary-care physician is uniquely placed to assess and address the impact of GERD on patients' lives. ( $\mathrm{J}$ Am Board Fam Pract 2005;18: 393-400.)

Gastroesophageal reflux disease (GERD) is a debilitating condition, characterized by symptoms of chronic, intermittent heartburn (a burning sensation in the chest and throat), and acid regurgitation (a sensation of acid in the esophagus or mouth), with esophagitis seen in a substantial minority of patients. ${ }^{1} \mathrm{Up}$ to $20 \%$ of the population is thought to be affected by at least weekly reflux symptoms, ${ }^{2}$ and it is estimated that GERD accounts for around $5 \%$ of a primary-care physician's workload. ${ }^{3}$

Submitted, revised, 11 May 2005.

From the David Geffen School of Medicine at UCLA, Beverly Hills, California (HL); Centre for Integrated Health Care Research, University of Durham, United Kingdom (PH); Outcomes Research, AstraZeneca R\&D, Mölndal, Sweden (IW); and Department of Public Health and Primary Health Care, The University of Bergen, Norway (IW)

Conflict of interest: HL has acted as an advisor to AstraZeneca, where proton pump inhibitors are manufactured for the treatment of GERD. PSH has acted as advisor and has received funding for research and academic activities from several gastrointestinal product companies, including AstraZeneca, Wyeth, Takeda, Altana, and GlaxoSmithKline. IW is employed by AstraZeneca.

Corresponding author: Harley Liker, MD, MBA, David Geffen School of Medicine at UCLA, 9675 Brighton Way Suite 350, Beverly Hills, CA 90210 (e-mail: hliker@mednet. ucla.edu).
GERD is a chronic disease, with over half of persons with weekly reflux symptoms affected for more than 5 years. ${ }^{4}$ In addition to the cardinal symptoms of heartburn and acid regurgitation that cause patients discomfort and pain, GERD is associated with a range of atypical symptoms of esophageal and extra-esophageal origin, including sleep disturbance, chest pain, asthma, chronic cough, and hoarseness. ${ }^{5}$ The impact of this host of symptoms and consequences on the everyday lives of patients with GERD is often overlooked. ${ }^{6}$

This paper will consider the current understanding of the impact of GERD on patients' healthrelated quality of life (HRQL), well-being, and work productivity from the primary care perspective. In contrast with recent reviews, ${ }^{7-9}$ we evaluate only data from studies of patients with GERD managed by the primary-care physician and surveys of individuals with GERD in the community.

\section{Methods}

We conducted a systematic review of the literature to identify research that addressed the impact of GERD on HRQL, well-being, and work produc- 
tivity in the primary-care or community setting. The Medline database was searched via PubMed, using the terms [reflux or GERD or GORD or esophagitis or oesophagitis or heartburn] and [quality of life or productivity or sleep], limited to articles in English that had abstracts and were published between January 1, 1965, and April 21, 2005. This search produced a total of 866 articles. The titles and abstracts of each study identified by the search were reviewed to identify articles that reported quality-of-life assessments conducted in patients with GERD consulting in primary care or in individuals with GERD symptoms drawn from the general population. Studies of GERD patients in secondary care or of GERD patients selected based on endoscopic findings were not included, as these groups are unlikely to be representative of the patient population seen by primary-care physicians. This strategy identified 9 articles included in this review. An additional 14 articles eligible for inclusion in this review were identified by handsearching the proceedings of recent conferences (Digestive Diseases Week and United European Gastroenterology Week) and from 2 recent comprehensive reviews of the HRQL impacts of GERD. ${ }^{7,9}$ These 23 articles were reviewed in full. The selection of studies for inclusion is outlined in Figure 1.

\section{Results}

What Is the Impact of GERD on HRQL?

One of the largest ever studies of the impact of GERD on HRQL, the ProGERD study, involved

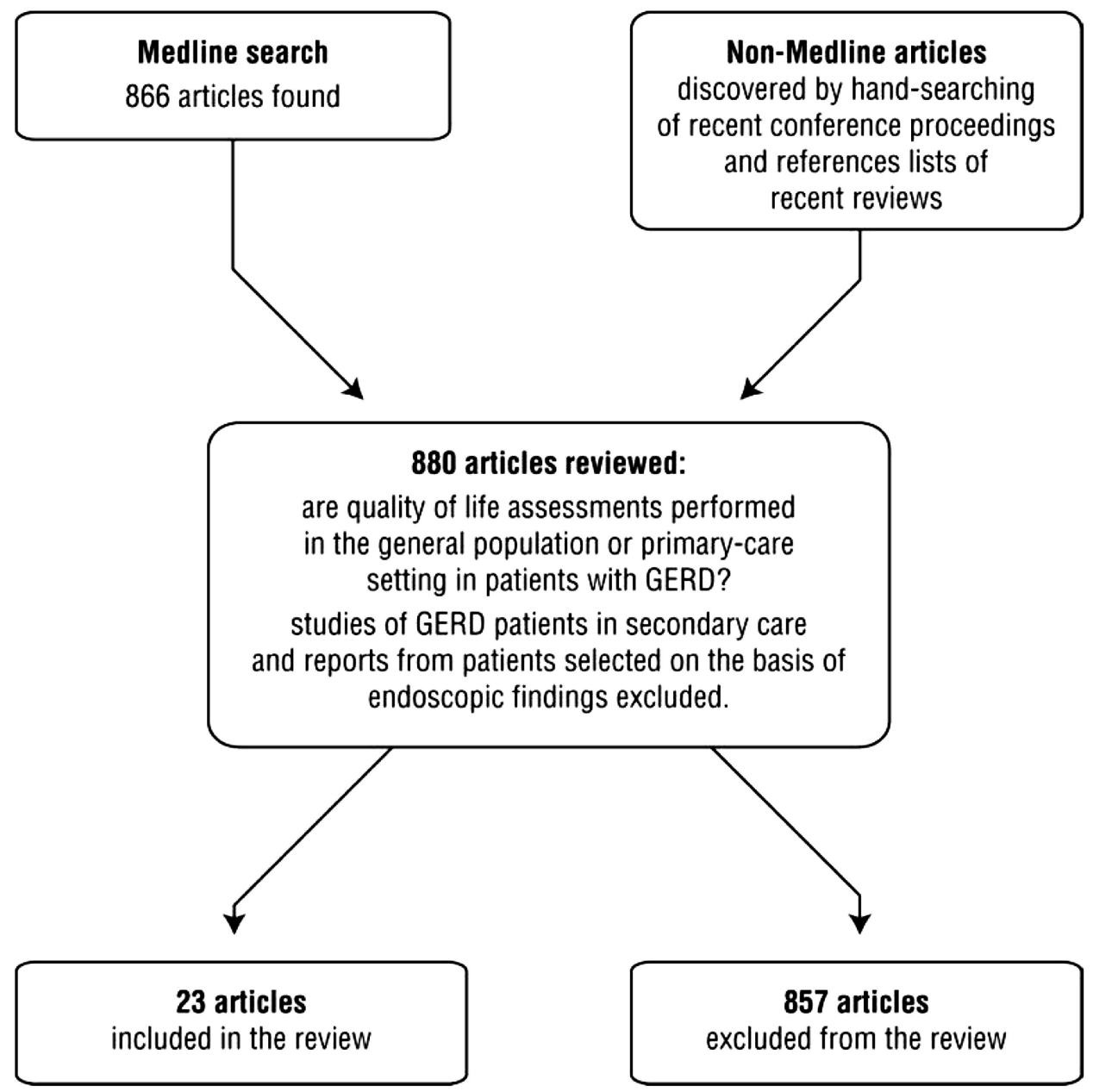

Figure 1. Flow diagram showing the selection of articles. The abstracts and titles of references within the Medline database were searched using PubMed with the terms (reflux or GERD or GORD or esophagitis or oesophagitis or heartburn and [quality of life or productivity or sleep]), and the search was limited to articles published in English with abstracts. 
over 6000 primary-care patients from Germany. ${ }^{10}$ Of this study population, 29\% had experienced reflux symptoms for more than 5 years, and $46 \%$ experienced symptoms daily. Using a validated generic instrument, the short form-36 (SF-36), it was evident that individuals with GERD had lower HRQL than that reported previously for the general German population, in terms of both physical and psychosocial aspects of well-being (Figure 2). Subjects with GERD had the greatest impairment in the domains of vitality, bodily pain, general health, physical and emotional role, and mental health and were also shown to be impaired in physical and social functioning. Indeed, the authors of this study concluded that GERD patients consulting in primary care experience lower HRQL than patients with other chronic diseases such as diabetes and cancer. Patients also completed a validated disease-specific instrument, the Quality of Life in Reflux and Dyspepsia questionnaire, which showed that patients experienced difficulties with eating/ drinking, sleep, physical/social functioning, emotions, and vitality as a result of their symptoms. The ProGERD study found that the impairments in HRQL observed were comparable whether or not patients with GERD had evidence of esophagitis or Barrett's esophagus. This study is likely to reflect the burden of illness in untreated patients consulting in primary care, because subjects were excluded if they had received continuous treatment with any acid-suppressant drug for more than 7 days in the 4 weeks before study entry.

The effect of GERD on HRQL was also investigated in a Swedish study of a representative sample of a predominantly rural population. ${ }^{11}$ Evidence from a random sample of 3000 individuals assessed using the SF-36 showed that subjects reporting GERD symptoms during the previous 3 months had significantly lower HRQL scores than people without such symptoms, whether or not esophagitis was present. As in the ProGERD study, this impairment was seen in both physical and emotional aspects of well-being.

\section{How Does GERD Impair HRQL?}

Large-scale population surveys provide some answers about the ways in which GERD and its associated symptoms impair HRQL. Although these have predominantly used nonvalidated questionnaires, they do provide detailed information on specific aspects of HRQL impairment in individuals with symptoms of GERD, including those who are not receiving medical advice for their condition.

A survey conducted by the National Heartburn Alliance in 2000 was mailed to over 750,000 US citizens and elicited responses from over 130,000 individuals with reflux disease, who provided feedback on 20 comprehensive, multipart questions on the subject of heartburn and its effects on normal life and functioning. ${ }^{12}$ Some $95 \%$ of respondents

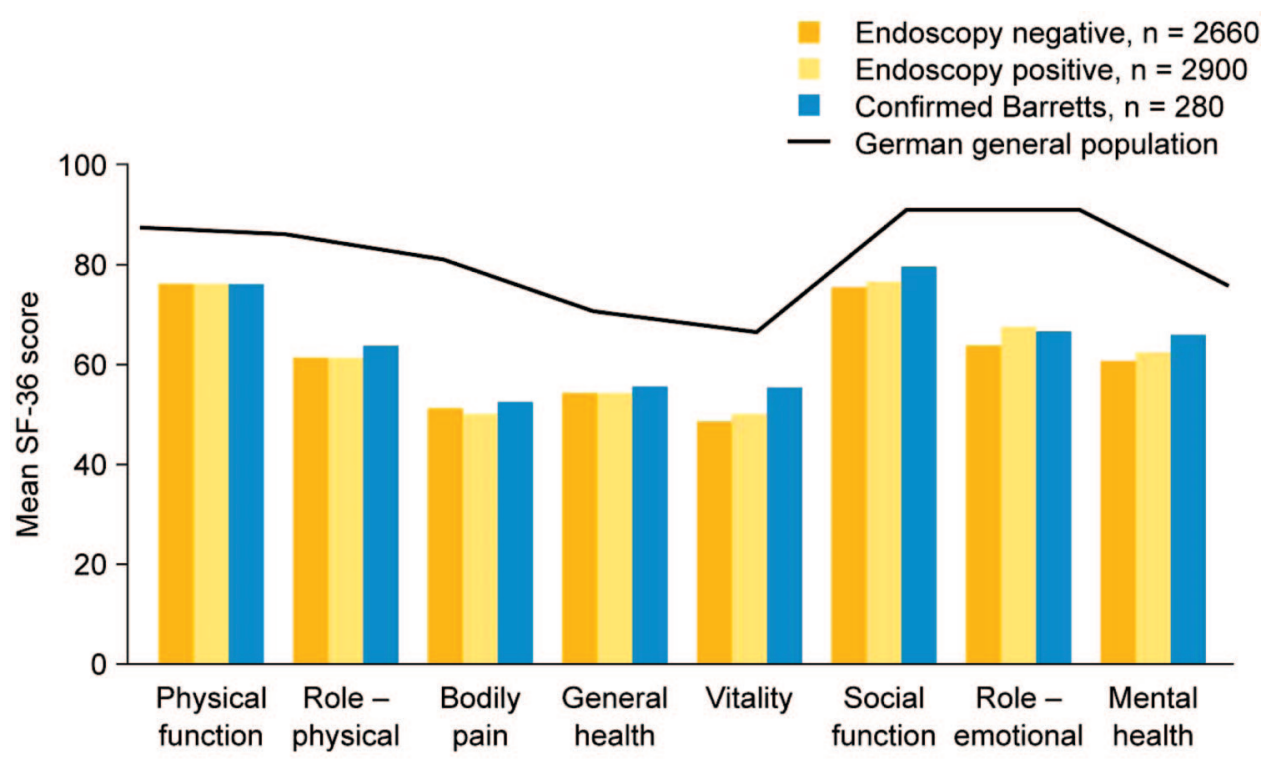

Figure 2. HRQL dimensions (assessed by SF-36) in German patients with reflux disease, compared with those of the German general population (adapted with permission from Kulig et al, $2003^{10}$ ). 
reported that they had had heartburn for more than 1 year and slightly more than half had experienced heartburn for more than 5 years. Almost $75 \%$ of respondents recorded heartburn at least twice a week and almost all respondents had heartburn at least once a month.

The survey found that heartburn severely limited daily activities and productivity. The majority of people reported a reduced enjoyment of food (more than 80\%); more than 60\% said that heartburn affected sleep, and over $40 \%$ found concentrating at work was difficult when heartburn occurred (Figure 3). Heartburn interfered with a wide range of daily activities, ranging from exercise, tending the garden, playing with children, and taking part in hobbies to enjoying intimacy and sex.

Another US survey that has provided insights into the wide-ranging effects of GERD on HRQL is the Gallup survey of 1000 adults experiencing heartburn at least once a week, conducted on behalf of the American Gastroenterology Association. ${ }^{13}$ Most respondents had experienced heartburn for over a year and $65 \%$ of respondents experienced both daytime and nighttime symptoms. Nocturnal heartburn was common, affecting nearly 8 of 10 respondents and contributing greatly to the impairment of subjects' well-being and function. More than $50 \%$ of those surveyed said that their heart-

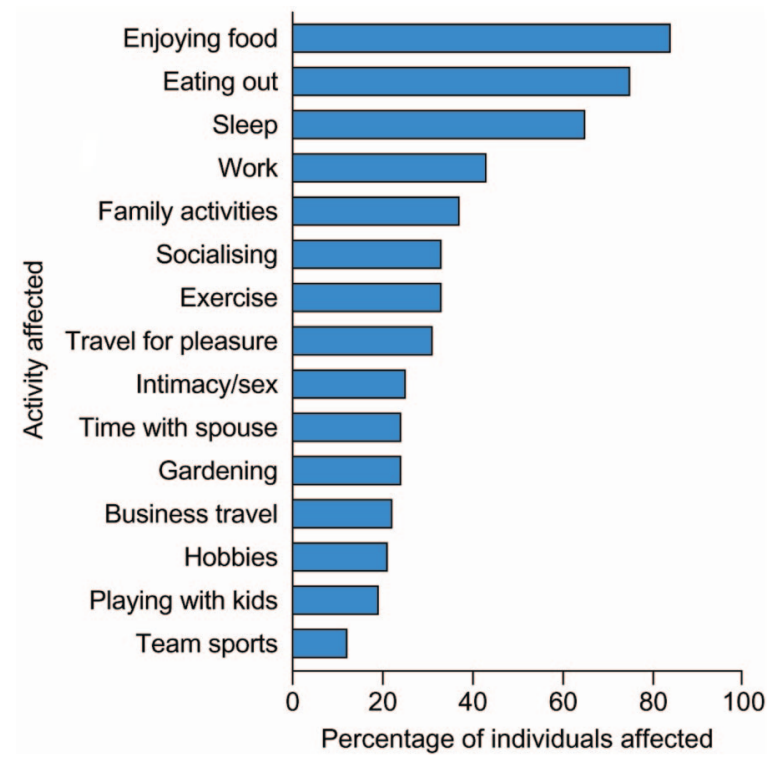

Figure 3. Activities affected by frequent heartburn among a community-based sample of 130,000 individuals experiencing heartburn in the United States. $^{12}$ burn affected them more at night than during the day; $63 \%$ reported an inability to get a good night's sleep and $40 \%$ found that daily function was compromised the day after nocturnal heartburn (Figure 4).

In a study of a random sample of adult primarycare patients conducted in Australia $(\mathrm{n}=400)$, it was found that $53 \%$ reported experiencing GERD, defined as heartburn, regurgitation of food and belching, chest pain, and unexplained cough. ${ }^{14}$ Of these subjects, $63 \%$ felt that their symptoms had a significant adverse effect on HRQL. In particular, subjects with GERD found that their symptoms were aggravated by housework (35\%), gardening (33\%), bending (22\%), and lifting (16\%). Sleep was disturbed for $57 \%$ of subjects and $55 \%$ reported that GERD made them irritable. Patients also reported that their enjoyment of meals was affected by GERD.

Thus the results of a number of surveys among the general population suggest that the chronic symptoms of GERD can have wide-ranging effects on HRQL. A systematic breakdown of the various parameters that comprise HRQL for patients in the community reveals that all measurements fall below the accepted level of normal among people suffering from GERD.

\section{Impact of GERD on Physical State}

Acute GERD symptoms are associated with considerable pain and discomfort. Heartburn may be worse when leaning forward or bending down, and almost half of GERD subjects report avoidance of any physical activities that might precipitate heartburn symptoms. ${ }^{15}$ In the Australian primary-care study described above, ${ }^{14}$ some $35 \%$ of respondents reported that regular housework activities precipitated heartburn and even slight exertion (for example, to bend and put on shoes) would cause heartburn in almost $25 \%$ of patients. Approximately one third of respondents reported restrictions in activities such as gardening, and $16 \%$ found their condition had an impact on sporting activities and exercise.

These findings are similar to those of the $\mathrm{Na}$ tional Heartburn Alliance survey, in which over $30 \%$ were concerned when exercising and over $20 \%$ of respondents found they were restricted when gardening. ${ }^{12}$ For the person with GERD, leisure activities may no longer be enjoyable and many sufferers avoid exercise and inadvertently 


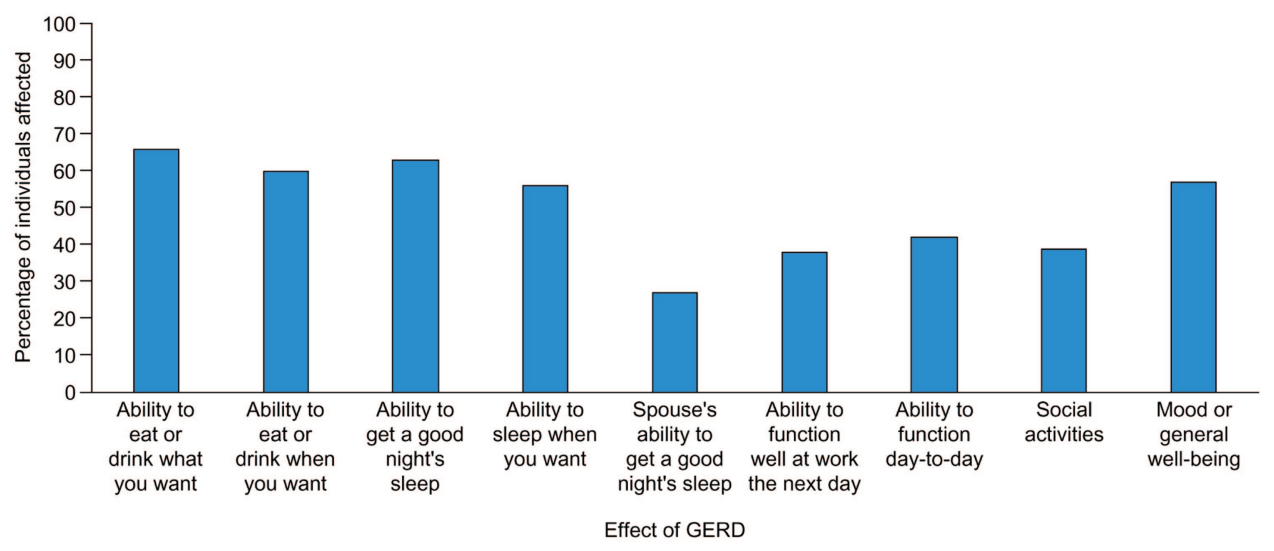

Figure 4. Effect of GERD on quality of life parameters in 1000 adult Americans experiencing heartburn at least once a week. ${ }^{13}$

adopt a sedentary lifestyle that can contribute to the worsening of their reflux symptoms.

\section{Impact on Emotional State}

Studies of patients with GERD suggest high levels of emotional distress, and patients often describe GERD in strong and emotive terms, conveying the extent to which this condition blights normal life. ${ }^{7,16}$ The sensitivity of these emotional aspects of HRQL to acid-inhibitory therapy supports the role of reflux symptoms as an important cause of patients' distress. ${ }^{17-19}$ A large number of subjects with GERD report impaired sexual activity and avoidance of intimacy because of their condition. In the National Heartburn Alliance survey, over 20\% of the 130,000 respondents felt that heartburn affected their sex lives. ${ }^{12}$ The American Gastroenterological Association survey of 1,000 subjects with frequent heartburn found that $56 \%$ of respondents believed their condition affected their mood and general well-being. ${ }^{13}$

Adding to the emotional distress caused by the painful and debilitating symptoms of GERD is the fact that many patients view heartburn symptoms as an indicator of generally poor health and frequently worry that their symptoms signify a serious underlying disease. In the primary care study conducted in Australia, ${ }^{14}$ over half of patients (52\%) were worried that their symptoms might be caused by a serious illness, and $55 \%$ said that GERD made them irritable. A survey of people in the community with symptoms of dyspepsia revealed that when a person consults their primary-care physician it is often because they harbor concerns that symptoms may be because of heart disease or cancer. $^{20}$

\section{Impact on Social Functioning}

Mealtimes are a major concern for many individuals living with GERD. Patients often feel that they cannot eat or drink normally, avoiding spicy or acidic foods and alcohol. They may find that this restriction in eating habits blights both daily enjoyment of food and social occasions where eating and drinking are a focus. In the American Gastroenterological Association survey, $31 \%$ of respondents reported that their social activities were affected by heartburn, ${ }^{13}$ and the survey conducted by the $\mathrm{Na}$ tional Heartburn Alliance showed that social function was widely affected by heartburn. ${ }^{12}$ Over $70 \%$ of respondents in the latter survey found that eating out was a problem, and almost $40 \%$ reported that heartburn affected family activities. Over $30 \%$ of respondents found social activities curbed by heartburn and one in 5 reported that time spent with their spouse or children was affected by heartburn.

\section{Impact on Sleep}

Disturbed sleep emerges as a very common occurrence in those with GERD and seems to have a wide-reaching effect on HRQL, mood, and patient functional ability. More than $60 \%$ of respondents to the American Gastroenterological Association survey reported that their sleep was disturbed by their GERD symptoms, with a subsequent impact on work performance and HRQL the next day. ${ }^{13}$ Among the subgroup of respondents reporting nocturnal heartburn (79\%), 75\% reported an effect 
on sleep. ${ }^{21}$ In particular, $63 \%$ of subjects with nocturnal heartburn could not sleep well and $40 \%$ reported that this affected their daily performance. These statistics suggest that well over $70 \%$ of people with frequent heartburn are troubled by nocturnal symptoms and reflect the results of an earlier US population-based survey, which noted significantly lower SF-36 subscale scores in individuals with nocturnal heartburn than in the otherwise healthy population $(P<.001) .^{22}$

A recent survey involving over 500 subjects with heartburn in the UK noted that $69 \%$ of subjects experienced nocturnal symptoms. ${ }^{23}$ Some $40 \%$ of those with nighttime heartburn experienced symptoms 2 to 7 nights each week, and $73 \%$ of those with nocturnal symptoms classed them as moderate to severe. Sleep was disturbed for $82 \%$ of those who had nocturnal heartburn, with $42 \%$ of subjects reporting that daily function was affected by disturbed sleep and 10\% resorting to sleep therapies in an attempt to manage this complaint.

Individuals with GERD may be particularly vulnerable to the effects of reflux during the night, when defense mechanisms are depressed. ${ }^{24}$ Nocturnal awakening in reaction to GERD probably has a protective effect, allowing subjects to clear acid from the upper gastrointestinal tract. Yet despite the body's attempts to deal with nocturnal reflux, some people with GERD experience acid regurgitation into the airways, resulting in chronic cough which may also keep the patient awake. ${ }^{25}$

\section{Impact on Productivity}

Studies in GERD cohorts and in managed care populations suggest high levels of lost work productivity because of GERD. Although typical GERD symptoms may cause patients to take time off for physician visits, often it is the combination of sleep disruption, lack of vitality, and restrictions in activity linked with GERD that account for productivity loss in work and during daily living. For example, a US household survey of people reporting chronic heartburn sampled a group of over 1000 employed persons to determine the impact of GERD on work productivity. ${ }^{26}$ This survey found that $2.6 \%$ of workers reported absenteeism because of GERD, whereas 33\% reported that GERD symptoms affected their productivity during working hours (presenteeism). The overall impairment of work productivity because of GERD was $6 \%$ and increased with worsening symptom severity. Thus, some $12 \%$ of subjects with mild symptoms reported their productivity was impaired by symptoms of reflux, compared with $40 \%$ of subjects with moderate symptoms and $48 \%$ of those with severe symptoms. Among this community-based sample of subjects with heartburn, younger employees and those experiencing nocturnal reflux symptoms reported the greatest reductions in work productivity.

Similarly, in a Swedish study designed to validate a GERD-specific work productivity and activity impairment tool, it was found that among 136 subjects visiting their primary-care physician with symptoms of GERD, work productivity was reduced by an average of $23 \% .{ }^{27}$ This reduction in productivity at work was accompanied by a $30 \%$ reduction in patient productivity while performing daily living activities, highlighting the impairment caused by GERD to both paid and unpaid work.

\section{Discussion}

GERD is a serious chronic disease that is generally managed by the primary-care physician. The pain and debility caused by the cardinal symptoms of heartburn and acid regurgitation disrupt many aspects of patients' lives and, together with associated symptoms and complications, adversely affect HRQL and productivity. People with GERD consistently experience lower HRQL than the general population and often exhibit HRQL impairments comparable with those seen in other chronic diseases such as asthma, heart failure, angina pectoris, and cancer. $^{7,28}$ Because heartburn is a painful symptom, it is not surprising that the most pronounced HRQL impairment was observed in the bodily pain area.

Pain is a key driver for consultation, and patients with upper gastrointestinal complaints typically consult because of the frequency or severity of their symptoms, particularly if their current therapy does not provide adequate relief. ${ }^{20,29,30}$ Patients also seek medical help because of fears and concerns that their reflux symptoms herald the onset of a serious and life-threatening disease, and this contributes to patients' anxiety and diminished HRQL. Whereas referral for endoscopy is necessary for patients with alarm symptoms, in their absence it is rarely required, and these patients can be treated effectively in primary care. ${ }^{31,32}$ As the symptom burden and HRQL impact of GERD is comparable in patients with and without esophagitis, endoscopy 
is of little benefit in the assessment and subsequent management of patients with uncomplicated GERD.

Importantly, it seems that effective treatment of GERD can help to reverse the damaging effects of GERD on HRQL. Studies conducted in the community setting have also shown that, by ameliorating reflux symptoms, acid inhibitory therapy significantly improves the emotional and psychosocial aspects, as well as physical aspects, of patients' HRQL. ${ }^{10,33-36}$ Improvements in sleep quality are thought to play a major role in these improvements, suggesting that active treatment of GERD may help to curb the vicious cycle of disturbed sleep patterns that can characterize chronic GERD and ameliorate the resulting problems of reduced productivity and impaired daily function.

Given the impact of GERD on HRQL, asking patients about the impact of their reflux symptoms may be useful during the diagnosis of GERD. ${ }^{37}$ Many patients are reluctant to discuss the full impact of their symptoms on their everyday lives and, in the absence of appropriate questioning, an inadequate picture may emerge. ${ }^{9,38}$ This can hinder appropriate management and result in potentially suboptimal treatment. ${ }^{39} \mathrm{We}$ offer an aide memoire to help evaluate the effects of GERD on HRQL in everyday clinical practice (Table 1). One outcome from a recent international meeting was agreement that symptoms occurring on 2 days each week are sufficient to meaningfully impair HRQL. ${ }^{40}$ Careful assessment of HRQL represents a valuable addi-

\section{Table 1. Proposed Checklist for Evaluating the Impact} of GERD

\footnotetext{
Physical activities and function

Impaired in sport and exercise

Daily activities limited (housework, bending, lifting)

Avoidance of exertion

Emotional and social well-being

Avoidance of social situations triggering heartburn

Food/alcohol restrictions

Impaired sexual function

Feelings of pain and discomfort leading to low mood

Worry over general health status (anxiety)

Productivity — work and daily living activities

Symptoms leading to time off work

Symptoms impacting on productivity at work

Avoidance of activities that precipitate symptoms

Nocturnal symptoms affecting next-day performance

Sleep disturbances

Nocturnal heartburn present

Nighttime cough or asthma

Sleep disturbed by symptoms

Impact on next-day well-being and functioning
}

tional approach to assist physicians in their evaluation and management of GERD.

We thank Winnie McFadzean, Dr. Becky Fox-Spencer, and Dr. Christopher Winchester for their editorial assistance with this manuscript, which was prepared with support from AstraZeneca.

\section{References}

1. Dent J, Brun J, Fendrick AM, et al. An evidencebased appraisal of reflux disease management-the Genval Workshop Report. Gut 1999;44 Suppl 2:S116.

2. Dent J, El-Serag HB, Wallander MA, Johansson S. The epidemiology of reflux disease: a systematic review. Gut 2005;545:710-7.

3. McCormick A, Fleming DM, Charlton J. Morbidity statistics from general practice: fourth national study 1991-1992. 1995; available: www.statistics.gov.uk/ downloads/theme_health/MB5No3.pdf. Accessed 28 Feb 2005.

4. Locke GR 3rd, Talley NJ, Fett SL, et al. Prevalence and clinical spectrum of gastroesophageal reflux: a population-based study in Olmsted County, Minnesota. Gastroenterology 1997;112:1448-56.

5. Hungin AP, Raghunath A, Wiklund I. Beyond heartburn: A review of the extra-oesophageal spectrum of reflux-induced disease. Fam Pract doi:10.1093/ fampra/cmi061. Epub 2005 July 15.

6. Webb DD. Introduction. GERD warrants increased physician appreciation and improved treatment. Postgrad Med 2001;Spec No:5-10.

7. Wiklund I, Talley NJ. Update on health-related quality of life in patients with gastroesophageal reflux disease. Expert Rev Pharmacoeconomics Outcomes Res 2003;3:341-50.

8. Kamolz T, Pointner R, Velanovich V. The impact of gastroesophageal reflux disease on quality of life: review of the literature. Surg Endosc 2003;17: $1193-9$.

9. Wiklund I. Review of the quality of life and burden of illness in gastroesophageal reflux disease. Dig Dis 2004;22:108-14.

10. Kulig M, Leodolter A, Vieth M, et al. Quality of life in relation to symptoms in patients with gastrooesophageal reflux disease - an analysis based on the ProGERD initiative. Aliment Pharmacol Ther 2003; 18:767-76.

11. Aro P, Ronkainen J, Storskrubb T, et al. Quality of life in a general adult population with gastroesophageal reflux symptoms and/or esophagitis: a report from the Kalixanda study. Gastroenterology 2003; 124(4 Suppl 1):A168.

12. National Heartburn Alliance. Survey 2000 results: a community perspective. 2000; available: http://www. heartburnalliance.org/survey2000.pdf. Accessed 14 Jul 2004.

13. American Gastroenterological Association. Under- 
standing heartburn in America. 2000; available: www. gastro.org/media/newsRelease00/studyNighttime. html. Accessed 18 Dec 2003.

14. Jasani K, Piterman L, McCall L. Gastroesophageal reflux and quality of life. Patient's knowledge, attitudes and perceptions. Aust Fam Physician 1999;28 Suppl 1:S15-8.

15. Talley NJ, Junghard O, Wiklund IK. Why do patients with gastroesophageal reflux disease (GERD) have a poor heath-related quality of life (HRQL)? Gastroenterology 2001;120(5 Suppl 1):A-423.

16. Grime J, Pollock K, Blenkinsopp A. Proton pump inhibitors: perspectives of patients and their GPs. Br J Gen Pract 2001;51:703-11.

17. Havelund T, Lind T, Wiklund I, et al. Quality of life in patients with heartburn but without esophagitis: effects of treatment with omeprazole. Am J Gastroenterol 1999;94:1782-9.

18. Ofman JJ. The economic and quality-of-life impact of symptomatic gastroesophageal reflux disease. Am J Gastroenterol 2003;98(3 Suppl):S8-14.

19. Talley NJ, Fullerton S, Junghard O, Wiklund I. Quality of life in patients with endoscopy-negative heartburn: reliability and sensitivity of disease-specific instruments. Am J Gastroenterol 2001;96: 1998-2004.

20. Lydeard S, Jones R. Factors affecting the decision to consult with dyspepsia: comparison of consulters and non-consulters. J R Coll Gen Pract 1989;39:495-8.

21. Shaker R, Castell DO, Schoenfeld PS, Spechler SJ. Nighttime heartburn is an under-appreciated clinical problem that impacts sleep and daytime function: the results of a Gallup survey conducted on behalf of the American Gastroenterological Association. Am J Gastroenterol 2003;98:1487-93.

22. Farup C, Kleinman L, Sloan S, et al. The impact of nocturnal symptoms associated with gastroesophageal reflux disease on health-related quality of life. Arch Intern Med 2001;161:45-52.

23. Hungin AP. Night time heartburn prevalence and impact: a telephone survey. Digestive Diseases Week, New Orleans (LA); 2004.

24. Orr WC. Sleep and gastroesophageal reflux: what are the risks? Am J Med 2003;115 Suppl 3A:109S$13 \mathrm{~S}$.

25. Orr WC. Sleep measures and gastroesophageal reflux in patients with symptomatic GERD. Am J Gastroenterol 2002;97(September Suppl):S27.

26. Dean BB, Crawley JA, Schmitt CM, et al. The burden of illness of gastro-oesophageal reflux disease: impact on work productivity. Aliment Pharmacol Ther 2003;17:1309-17.

27. Wahlqvist P. Symptoms of gastroesophageal reflux disease, perceived productivity, and health-related quality of life. Am J Gastroenterol 2001;96(8 Suppl): S57-61.
28. Revicki DA, Wood M, Maton PN, Sorensen S. The impact of gastroesophageal reflux disease on healthrelated quality of life. Am J Med 1998;104:252-8.

29. Crawley JA, Hamelin B, Gallagher E. Does it matter why heartburn sufferers seek health care?. Gastroenterology 2000;118:A209.

30. Delaney BC. Why do dyspeptic patients over the age of 50 consult their general practitioner? A qualitative investigation of health beliefs relating to dyspepsia. Br J Gen Pract 1998;48:1481-5.

31. Heidelbaugh JJ, Nostrant TT, Kim C, Van Harrison R. Management of gastroesophageal reflux disease. Am Fam Physician 2003;68:1311-8.

32. Dent J, Jones R, Kahrilas P, Talley NJ. Management of gastro-oesophageal reflux disease in general practice. BMJ 2001;322:344-7.

33. Leodolter A, Kulig M, Nocon M, et al. Esomeprazole therapy improves sleep disorders in patients with gastroesophageal reflux disease (GERD): a report from the ProGerd study. Gastroenterology 2003;124(Suppl 1):A226.

34. Johnson DA, Orr W, Marple C, et al. Effect of esomeprazole on sleep quality in patients with GERD: patient-reported outcomes from a randomized controlled trial using the Pittsburgh Sleep Quality Index (PSQI) questionnaire. Gastroenterology 2004;126(4 Suppl 2):A601-602.

35. Johnson DA, Orr W, Cuccia A, et al. Esomeprazole for the relief of moderate to severe nighttime heartburn and associated sleep disturbance in patients with GERD: a multicenter, randomized, doubleblind, placebo-controlled, 4-week study. Gastroenterology 2004;126(4 Suppl 2):A336.

36. Carlsson R, Dent J, Watts R, et al. Gastro-oesophageal reflux disease in primary care: an international study of different treatment strategies with omeprazole. International GORD Study Group. Eur J Gastroenterol Hepatol 1998;10:119-24.

37. Gralnek IM. Diagnosis and management of gastroesophageal reflux disease in the primary care setting: can health-related quality of life play a role? Am J Gastroenterol 2001;96(8 Suppl):S54-6.

38. McColl E, Junghard O, Wiklund I, Revicki D. Assessing symptoms in gastroesophageal reflux disease: how well do clinicians' assessments agree with those of their patients?. Am J Gastroenterol 2005;100: 11-8.

39. Crawley JA, Schmitt CM. How satisfied are chronic heartburn sufferers with their prescription medications? Results of the patient unmet needs survey. J Clin Outcomes Management 2000;7:29-34.

40. Dent J, Armstrong D, Delaney B, et al. Symptom evaluation in reflux disease: workshop background, processes, terminology, recommendations, and discussion outputs. Gut 2004;53 Suppl 4:iv1-24. 